\title{
Real-life experience with personally familiar faces enhances discrimination based on global information
}

Meike Ramon, Goedele Van Belle

Despite the agreement that experience with faces leads to more efficient processing, the underlying mechanisms remain largely unknown. Building on empirical evidence from unfamiliar face processing in healthy populations and neuropsychological patients, the present experiment tested the hypothesis that personal familiarity is associated with superior discrimination when identity information is derived based on global, as opposed to local facial information. Diagnosticity and availability of local and global information was manipulated through varied physical similarity and spatial resolution of morph faces created from personally familiar, or unfamiliar faces. We found that discrimination of subtle changes between highly similar morph faces was unaffected by familiarity. Contrariwise, relatively more pronounced physical (i.e., identity) differences were more efficiently discriminated for personally familiar faces, indicating more efficient processing of global, as opposed to local facial information through real-life experience. 
1 Real-life experience with personally familiar faces enhances discrimination

2 based on global information

3

4

5

6

7

8

9 Université de Fribourg

10 Faucigny 2

111700 Fribourg

12 Switzerland

13 email: meike.ramon@unifr.ch
Meike Ramon ${ }^{1} \&$ Goedele Van Belle ${ }^{2}$

${ }^{1}$ University of Fribourg, Switzerland

${ }^{2}$ University of Louvain, Louvain-la-Neuve, Belgium

14

15 


\section{Abstract}

17 Despite the agreement that experience with faces leads to more efficient processing, the underlying 18 mechanisms remain largely unknown. Building on empirical evidence from unfamiliar face processing in

19 healthy populations and neuropsychological patients, the present experiment tested the hypothesis that

20 personal familiarity is associated with superior discrimination when identity information is derived based

21 on global, as opposed to local facial information. Diagnosticity and availability of local and global

22 information was manipulated through varied physical similarity and spatial resolution of morph faces

23 created from personally familiar, or unfamiliar faces. We found that discrimination of subtle changes

24 between highly similar morph faces was unaffected by familiarity. Contrariwise, relatively more

25 pronounced physical (i.e., identity) differences were more efficiently discriminated for personally familiar

26 faces, indicating more efficient processing of global, as opposed to local facial information through real-

27 life experience.

28 Keywords: Face processing, personal familiarity, real-life exposure, global information integration 


\section{Introduction}

Humans are highly efficient at processing faces of conspecifics. Within a few hundred milliseconds, we can categorize faces according to their gender, expression, race and familiarity, as well as identify them. The social importance of face processing, and its complexity as a perceptual and cognitive process has motivated numerous investigations of the underlying mechanisms. Several lines of research indicate that processing of identity information is linked to observers' ability to integrate global facial information, also referred to as holistic processing, a hallmark of adult's face processing expertise (McKone, Kanwisher, \& Duchaine, 2007; Mondloch, Pathman, Maurer, Le Grand \& de Schonen, 2007; Richler \& Gauthier, 2013). Neuropsychological studies have demonstrated that face processing deficits observed in prosopagnosia can be accounted for by patients' impairment of holistic processing (e.g., Ramon, Busigny \& Rossion, 2010; for a review see Rossion, 2014). Furthermore, recent evidence suggests a direct relation between the extent of holistic processing and healthy observers' face processing efficiency (Wang et al., 2012).

While the most impressive demonstration of humans' face processing ability is observed for familiar faces, the bulk of empirical evidence regarding its determinants stems from experiments involving unfamiliar face stimuli. Several studies indicate that personally familiar faces are processed more efficiently than their unfamiliar counterparts (e.g., Bruce, Henderson, Greenwood, Hancock, Burton \& Miller, 1999; Burton, Wilson, Cowan \& Bruce, 1999; Ramon, Caharel \& Rossion, 2011). Familiar identities can be behaviorally categorized as such significantly faster than unfamiliar faces, within as little as 310 ms (Ramon, Caharel \& Rossion, 2010; see also Busigny, Bled, Besson \& Barbeau, 2012), with differential electrophysiological responses occurring 100 ms prior (Caharel, Ramon \& Rossion, 2014). Moreover, the presence of an underlying facial representation stored in memory makes perceptual processing more robust to variations in the input characteristics. For instance, we can easily recognize a famous or personally familiar face based on their eyes alone (Sadr, Jarudi \& Sinha, 2003; Ramon, Busigny, Gosselin \& Rossion, submitted), or from a highly degraded image (Watier \& Collin, 2009; 
55 Loftus \& Harley, 2005; Ramon et al., submitted), and even after considerable time periods (Bahrick,

56 Bahrick \& Wittingler, 1975). Contrariwise, identity processing in unfamiliar faces is highly dependent on

57 the visual characteristics of the stimulus input, i.e., availability of color, texture, and surface reflectance

58 (Russell, Sinha, Biederman \& Nederhouser, 2006; Jiang, Blanz \& Rossion, 2011), and is hence more

59 prone to error given superficial image variations (e.g., viewpoint or image quality; Bruce 1986; Roberts

60 and Bruce 1989; Bruce, Henderson, Newman \& Burton, 2001).

Together, these observations support the general consensus that unfamiliar and personally familiar faces are processed differently (cf., Tong \& Nakayama, 1999; Megreya \& Burton, 2006; Carbon, 2008;

Gobbini et al., 2013). However, there is little understanding of the underlying mechanisms promoting such differential processing. Early studies using famous faces have suggested that familiarity affects processing of internal facial information in general (Ellis, Shepherd \& Davies, 1979; Young, Hay, McWeeny, Flude \& Ellis, 1985; Brooks \& Kemp, 2007). Other investigations have provided inconsistent results regarding whether or not the effects of familiarity are restricted to processing of the eyes (O’Donnell \& Bruce, 2001), or extend to the less salient mouth region (Barton, Radcliffe, Cherkasova, Edelman \& Intriligator, 2006; Van Belle, Ramon, Lefèvre \& Rossion, 2010). However, more recent findings may reconcile these seemingly conflicting findings. Ramon $(2015 \mathrm{a}, \mathrm{b})$ reported that personal familiarity affects discrimination of vertical displacements of the eyes and the mouth, as well as changes of the overall configuration between these two sources of information. These findings suggest that personal familiarity may not affect processing of specific types of facial information, but rather modulate the processing style engaged in.

The present study sought to extend these previous findings by varying the degree to which local or global information was diagnostic for identity discrimination. The underlying idea is that personal familiarity facilitates perceptual processing — and thus discrimination —of faces through the presence of a 
80 processing for familiar compared to unfamiliar faces would be observed along with decreased reliance on

81 local, piecemeal information.

In a delayed matching paradigm, observers performed forced-choice decisions of facial identity.

83 The face stimuli used to this end were derived from morph continua similar to those used in investigations

84 of categorical perception of identity, or expression (Beale and Keil, 1995 ; Gilaie-Dotan \& Malach, 2005;

85 Fox, Moon, Iaria \& Barton, 2009; Ramon, Dricot \& Rossion, 2010). The parametric variations in identity-

related physical information offered a means to manipulate the perceptual similarity and hence ambiguity

of information supporting discriminative decisions. ${ }^{1}$ While dissimilar stimuli could be more easily

discriminated based on global information, discrimination of more similar ones would be comparably less

efficient and require more local distinctive feature sampling. Hence, our experimental conditions differed

in the extent to which discrimination would be based on global, or holistic processing (defined here as the

fast and automatic process leading to an internal representation of the face as a whole; Galton, 1883;

92 Rossion, 2013).

Importantly, to investigate whether personal familiarity selectively affects global processing, or

leads to a general processing advantage, two groups of observers completed the same task. Control

subjects were unfamiliar with all of the identities depicted. The experimental group comprised subjects who were highly personally familiar with half of the identities, which represented their classmates.

Thus, contrary to previous studies, we varied two aspects that are considered to modulate observers' face processing efficiency. On the one hand, the physical similarity of face stimuli (which covaries with discrimination efficiency based on global information), as well as observers' familiarity with face stimuli. In line with the high discrimination performance reported for familiar vs. unfamiliar face

101 discrimination, a beneficial effect of familiarity was anticipated for conditions of low similarity

\footnotetext{
${ }^{1}$ Previous studies have demonstrated that increased physical similarity is associated with decreased discriminability and therefore more piecemeal processing of local cues (e.g. pixel-based intensity, or color differences; Barton et al., 2006; Orban de Xivry, Ramon, Lefèvre \& Rossion, 2008; Busigny, Graf, Mayer \& Rossion, 2010; Van Belle, Lefèvre \& Rossion, 2012), i.e. decreased reliance on initial holistic or global processing. Note that other authors have applied the same morphing techniques to e.g. identities differing merely in regard to a single feature (Goffaux, 2012) or a metric relation between features (Gilad-Gutnik, Yovel \& Sinha, 2012).
} 
102 considered to involve reliance on global information. Contrariwise, no such familiarity-related advantage

103 was expected for discrimination of highly similar faces, which would rely on observers' ability to identify

104 locally circumscribed details.

105 Naturally, local details are also available in conditions of low similarity, and thus could be used for

106 face discrimination. Therefore, we incorporated a third condition involving low similarity. Here, the local,

107 high resolution information was made unavailable through stimulus blurring (Sergent, 1986; Collishaw \&

108 Hole, 2000; Schwaninger, Wallraven, Cunningham \& Chiller-Glaus, 2006; Gilad-Gutnick et al., 2012).

109 Thus, we removed high spatial frequency information typically used for piecemeal analytic processing

110 (Goffaux, 2009; Goffaux \& Rossion, 2006).

111 In sum, we manipulated stimulus similarity, availability of high resolution local details, as well as

112 personal familiarity to directly test - for the first time to our knowledge — the hypothesis that familiarity

113 leads to an advantage in global/holistic, but not local processing. Two possible outcomes were

114 anticipated. First, if familiarity is associated with an advantage at processing local discriminative

115 information, we should observe a familiarity advantage across both conditions involving high resolution

116 images enabling the use of said information. Alternatively, if familiarity is advantageous for automatic

117 global processing, we should observe an experience-related benefit for conditions involving low stimulus

118 similarity - regardless of whether high spatial information used for local information processing is

119 available.

120 Methods

121 Procedure and apparatus

122 Participants performed a two-to-one alternative forced-choice delayed matching task. Each trial

123 started with a centrally presented fixation cross. Upon its fixation, two probe faces (distance between the

124 inner borders: $5^{\circ}$ of visual angle) were presented side by side for $2.5 \mathrm{~s}$, during which they could be

125 explored freely. Immediately thereafter the probe faces were replaced by a single centrally presented test 
126 face, which was identical to one of the two probes (sides counterbalanced). The test face remained on

127 screen until participants indicated to which of the probes it corresponded by pressing the corresponding

128 left or right arrow button on the keyboard. The next trial was initiated immediately after each response.

129 The experiment consisted of six blocks, with each block comprising 40 trials; three blocks

130 involved presentation of familiar and unfamiliar faces, respectively. Each block consisted of trials of one

131 of the three experimental conditions (Full 20\%, Full 50\%, Blur 50\%; see below). The order of the blocks

132 in terms of familiarity and experimental condition was counterbalanced across participants (note that

133 control subjects were unfamiliar with all faces used as stimuli; see below). Within each block there was

134 an equal amount of left and right correct response sides and both faces from each of the 10 stimulus pairs

135 appeared twice as test stimuli. This procedure ensured equal likelihood of perceiving either of the

136 identities of a given morph continuum (see Ramon et al., 2010).

137 To become familiar with the task, participants completed five practice trials prior to the main

138 experiment. These practice trials contained faces that were not used in the main experiment, and were

139 excluded from analysis. Stimuli were displayed using Presentation or Eprime software, on a 22" Sony

140 Trinitron monitor (58cm viewing distance, $1400 \times 1050$ pixel resolution, $85 \mathrm{~Hz}$ refresh rate). Probe and

141 test faces' height comprised 10.3 and $11.5^{\circ}$ of visual angle, respectively. This roughly corresponds to the

142 size of a real face viewed at normal conversational distance of $90 \mathrm{~cm}$ (Hall, 1966). Both stimulus display

143 and response registration was controlled by an Intel Centrino vPro.

\section{Stimuli}

Two different sets of stimuli were used in the main experiment. The first was taken from a previous study (Ramon et al., 2010) and involved 10 morph continua, the extremes of which were unfamiliar to all participants. An additional set of 10 morph continua were created between pairs of faces with which half of the participants were personally familiar: their classmates. To this end full-front

149 photographs of 26 students of the same class were taken under identical conditions (distance, lighting).

150 The photographs of five male and one female student were excluded from the final set of (Caucasian, 
151 female) familiar faces used, due to the presence of facial hair or make-up at the time the photographs

152 were taken. Using Adobe Photoshop, the remaining 20 familiar face stimuli were cropped of external

153 features and hair (see Figure 1a) and morph continua were created with Photo Morpher v3.10 (Morpheus,

154 Santa Barbara, CA, USA) following the same procedure as used by Ramon et al. (2010). Specifically,

155 face pairs were selected based on eye color, shape and overall luminosity (average pixel intensity) of the

156 face. For each face, 350 points were placed on the critical features (encompassing the pupils, iris, eye

157 bulbs, eye lids, eye brows, mouth, nose and overall facial contour) to allow smooth transitions between

158 the stimuli created per morph continuum (two original faces representing the extremes, with $10 \%$

159 increments; see Figure 1a).

a.
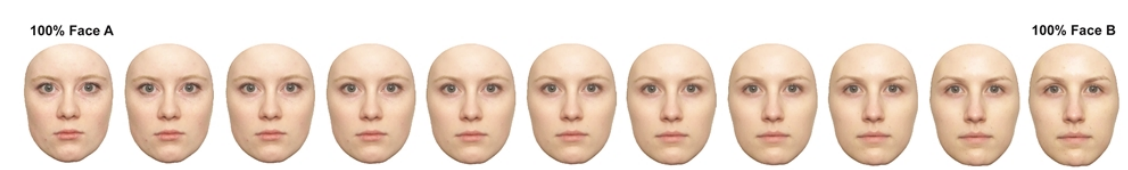

b.
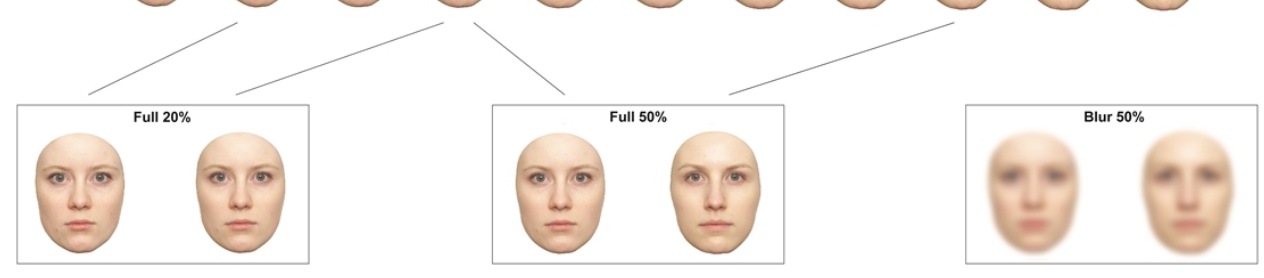

Figure 1. Stimuli discriminated in the delayed matching task. a. An example of a familiar face morph continuum. Unfamiliar stimuli were those created and used in a previous study (see Ramon et al., 2010 for examples and details regarding stimulus creation), which were unfamiliar to all subjects tested. Familiar morph continua were created from pairs of classmates of experimental subjects tested, and were unfamiliar to control subjects. b. Examples of stimulus pairs to be discriminated in the 2AFC delayed matching task (pairs were taken from either side of a continuum).

As described above, our hypothesis was that personal familiarity is associated with facilitated

172 (i.e., the point where both identities would be perceived with equal likelihood; see e.g. Beale \& Keil,

1742005 ; note that as here we were not interested in testing categorical perception, but the effects of stimulus 
175 similarity, the categorical boundary was considered as the midpoint of the morph continua and was hence

176 not individually defined). The second condition involved pairs of stimuli, which differed by $50 \%$ (i.e.,

177 physically dissimilar pairs, Full 50\%) and were located on opposite sides of the categorical boundary of

178 the morph continua. Note that the distance of these more dissimilar items relative to both extremes (i.e.,

179 original faces), and the categorical boundary was identical to the physical difference between Full 20\%

180 pairs (see Figure 1). ${ }^{2}$ In the Full $20 \%$ condition the faces are more similar than in the Full 50\% condition,

181 making the comparison more ambiguous, and comparably more dependent on local information (see e.g.,

182 Barton et al., 2006). A third condition involved the same dissimilar pairs (i.e., those used in the Full 50\%

183 conditions), to which a Gaussian blur (30 pixel radius; see e.g., Gilad-Gutnik et al., 2012) was applied to

184 make high-resolution information (e.g., freckles, wrinkles, etc.) unavailable. These physically dissimilar

185 pairs of blurred faces (Blur 50\%) comprised the third experimental condition, created to disable to the use

186 of local features in the low similarity condition.

\section{Participants}

Twelve participants (mean age: $23 \pm 1 ; 3$ male), who were personally familiar with half of the

individuals depicted in the stimuli (from here on referred to as participants comprising the 'experimental group'), were financially compensated for their participation. They were all senior year psychology master students who had been following classes in the same group of $\sim 30$ students for about two years at the time of testing; some knew each other for a maximum of 5 years (data collection occurred while

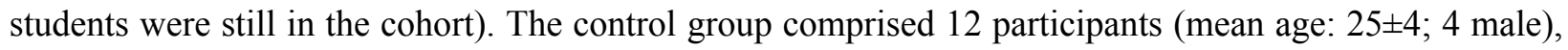

194 who were unfamiliar with all individuals' images used to create the face stimuli, and were also financially compensated for their participation. The experiments were undertaken with the understanding and written consent of each subject, and conform to The Code of Ethics of the World Medical Association

197 (Declaration of Helsinki).

\footnotetext{
${ }^{2}$ For trials on which probe stimuli differed by $20 \%$ (Full $20 \%$, see Figure 1a), probes were selected to depict a given identity by $90 \%$ and $70 \%$ (test stimuli always depicted a given identity by $90 \%$ ). For trials on which probe stimuli differed by $50 \%$ (Full $50 \%$, Blurred $50 \%$ ), the probes represented a given identity by $70 \%$ and $20 \%$; test stimuli always depicted a given identity by $20 \%$.
} 
198

199

200

201

202

203

204

205

206

207 208

\section{Analyses and results}

The analyses were conducted separately on accuracy scores and correct RTs as individual subjects may differ in terms of the measure they exhibit performance differences (Pachella, 1974; Meyer, Irwin, Osman \& Kounios, 1988). Raw accuracy and RT values per group and condition, as well as 95\% bootstrapped confidence intervals can be found in the supplemental material (Table S1). None of the conditions were associated with chance, or ceiling level performance; outliers (trials in which the RT exceeded the average RT +/- 2SD per condition and subject) were removed from the data.

To test the hypothesis that familiarity affects performance differently across conditions, Generalized Estimating Equations were applied to test for a three way interaction in a repeated measures model with group (experimental vs. control) as a between-subjects factor, and condition (Full 50\%, Full $20 \%$, Blur $50 \%$ ) and familiarity of stimuli (familiar vs. unfamiliar) as within-subjects factors. Using a binomial logit link distribution for accuracy, and a normal distribution for RT, we observed a significant three-way interaction for accuracy scores $\left(\right.$ Wald $\left.\mathrm{Chi}^{2}(2)=13.82, p<.01\right)$, but not for correct RTs (Wald $\left.\operatorname{Chi}^{2}(2)=3.36, p=.19\right)$.

To further investigate this interaction, we performed Bonferroni corrected post-hoc contrasts between individual factor level combinations. To facilitate the interpretation of these contrasts, they were performed on familiarity indices computed for each subject and condition ((familiar - unfamiliar) / (familiar + unfamiliar)). These familiarity indices capture potential effects of stimulus familiarity and will be referred to as 'the familiarity advantage' in the following. Group means of the familiarity advantage for accuracy across conditions are displayed in Figure 2. 


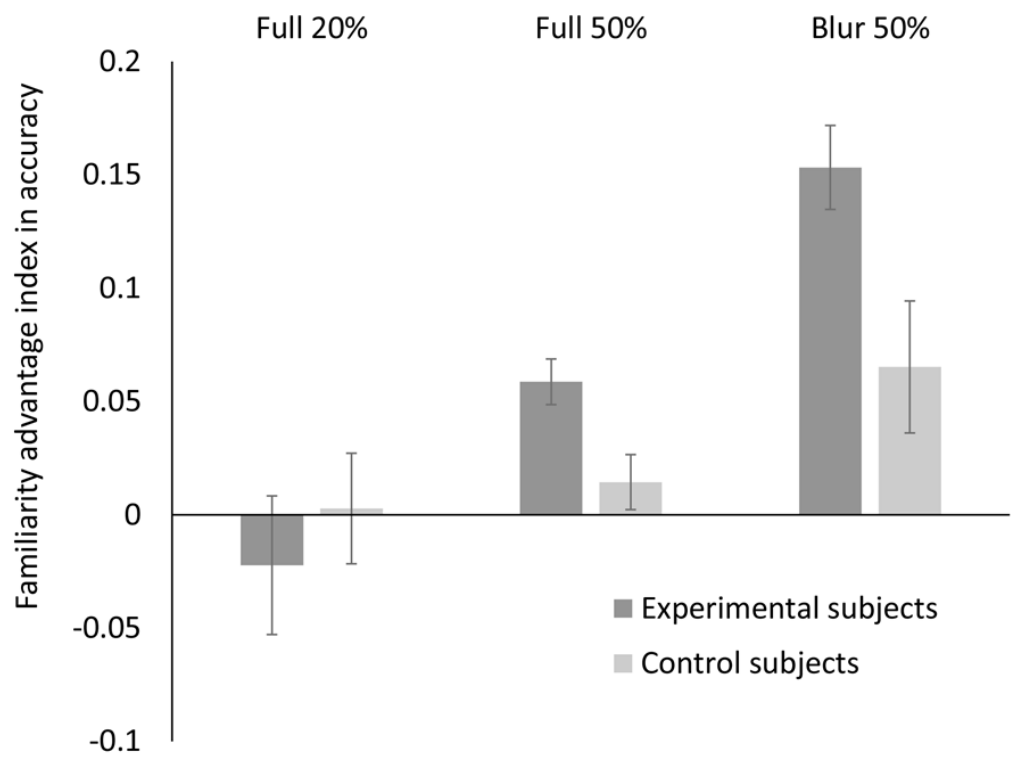

Figure 2. Familiarity advantage in the $2 \mathrm{AFC}$ delayed matching task with personally familiar and unfamiliar morph stimuli. Mean familiarity advantage ((familiar - unfamiliar) / (familiar + unfamiliar)) for accuracy scores per condition observed for control, as well as experimental subjects. Error bars represent standard errors for both measures. Note that for control subjects, all faces presented were unfamiliar.

T-tests revealed that the familiarity advantage was larger for the experimental group than for the control group in the Full $50 \%$ condition $(\mathrm{t}=2.80, p<.05)$ and in the Blur $50 \%$ condition $(\mathrm{t}=2.55, p<.05)$, but not in the Full $20 \%$ condition $(\mathrm{t}=.64, p=.53)$. Furthermore, in the experimental group the familiarity advantage was significantly smaller in the Full $20 \%$ than in the Full $50 \%(\mathrm{t}=2.73, p<.05)$ condition, and even larger in the Blur 50\% $(\mathrm{t}=3.98, p<.01)$ than the Full $50 \%$ condition. In the control group, the difference between familiar and unfamiliar stimuli did not significantly vary across conditions ( $p s>.05)$.

\section{Discussion}

Several lines of empirical evidence suggest a relationship between face processing efficiency and

231 the ability to rapidly integrate information from across the entire face into a unified percept, also referred

232 to as holistic processing. Some studies indicate a direct association between the degree of holistic

233 processing exhibited by healthy observers, and the efficiency with which upright faces are processed (e.g.,

234 Wang et al., 2012). Experimental manipulations utilized to disrupt holistic processing include stimulus inversion, as well as increased physical stimulus similarity. Both lead to reliable decreases in face 
237 style (Barton et al., 2006; Orban de Xivry et al., 2008, Van Belle, De Graef, Verfaillie, Busigny \& 238 Rossion, 2010), a phenomenon also observed in patients with prosopagnosia (i.e., the face selective 239 recognition deficit due to brain damage), who have lost the ability to process faces holistically (e.g., 240 Bukach, Le grand, Kaiser, Bub \& Tanaka, 2008; Busigny \& Rossion, 2010; Ramon et al., 2010a; Van 241 Belle, Busigny, Lefèvre, Joubert, Felician, Gentile, Rossion, 2011; Van Belle, De Graef, Verfaillie, 242 Busigny \& Rossion, 2010; Van Belle, Lefèvre, Laguesse, Busigny, De Graef \& Verfaillie, 2010; Rossion, 243 2014).

The present study aimed to investigate the effect of repeated, real-life experience with personally

245 familiar individuals on perceptual processing of faces. Naturally, healthy observers have no difficulty in 246 determining the identity of familiar individuals (see also e.g., Jenkins, White, Van Montfort \& Burton, 247 2011; Ramon et al., submitted) - a task for which ceiling effects can be expected. To manipulate the 248 relative reliance on global versus local information processing for face discrimination, the stimulus 249 material used here involved morph faces of varied physical similarity (e.g., Beale \& Keil, 1995; Gilaie250 Dotan \& Malach, 2007; Ramon et al., 2010b; Rotshtein et al., 2005). Following previous research, 251 irrespective of familiarity, discrimination of highly similar faces was assumed to be less efficient and rely 252 more on processing of local details (Barton et al., 2006; Orban de Xivry et al., 2008). Contrariwise, 253 dissimilar faces were anticipated to be distinguished more efficiently given automatic extraction of global 254 information from across the entire face. individuals used to create the morph stimuli presented. That is, not only did the face stimuli differ in their respective physical similarity, but also regarding the presence of a facial representation stored in memory. Replicating previous findings (e.g., Bruce et al., 1999; Burton et al., 1999; Ramon et al., 2011; Ramon, 2015a,b), we found that personal familiarity was associated with enhanced face discrimination performance. Using morph stimuli differing in physical similarity and subjects' familiarity, we sought to 
262 potential outcomes were anticipated. Increased performance for familiar versus unfamiliar faces for high

263 resolution images only (i.e., irrespective of physical (dis)similarity) would indicate higher efficiency at

264 discriminating faces based on local information. Alternatively, a familiarity-dependent advantage for

265 discrimination of dissimilar faces only (i.e., for both high resolution and blurred images) would support

266 the idea of experience-related increased efficiency for discerning facial identity changes based on global

267 information.

268 The performance profiles observed for the discrimination of morph stimuli created from 269 personally familiar faces was markedly different from that of unfamiliar faces. First, discrimination of 270 highly similar (Full 20\%) face morphs, which highly relies on local/featural processing (e.g., Barton et 271 al., 2006; Orban de Xivry et al., 2008), was unaffected by familiarity. This finding, which cannot be 272 accounted for in terms of floor effects, indicates that familiarity, i.e., extensive prior real-life experience, 273 does not lead to more proficient performance when processing relies on local information. Note that this 274 coincides with Barton et al.'s (2006) findings that higher ambiguity leads to an increase in difficulty of 275 perceptually based decisions, as well as the need to accumulate more data (in their study: more fixations, 276 longer durations; see also Althoff \& Cohen, 1999). Second, mirroring the high efficiency with which 277 personally familiar faces are generally processed, performance increased when discrimination of high278 resolution, dissimilar faces (Full 50\%) was required. Moreover, performance at discriminating the same

279 level of similarity was superior for familiar relative to unfamiliar stimuli despite the unavailability of 280 high-resolution, local information (Blur 50\%). These results are a clear indication that facial 281 representations stored in memory, as is the case for personally familiar faces, facilitate global processing 282 and reduce reliance on high-resolution local information for face discrimination.

283 Increased holistic processing of personally familiar faces? could be considered to tap into holistic processing, the present findings would support the notion that 286 visual experience modulates holistic processing (see e.g., Rossion, 2013). Previous studies exploring the 
287 effects of cross-cultural and cohort-dependent exposure have reported increased holistic processing and 288 superior face discrimination for faces with which subjects had extensive exposure, e.g., own-race (Michel, 289 Rossion, Han, Chung \& Caldara, 2006; Michel, Corneille \& Rossion, 2007), own-age faces (de Heering 290 \& Rossion, 2008; Kuefner, Macchi Cassia, Picozzi \& Bricolo, 2008), and faces presented in their 291 canonical orientation (Van Belle et al., 2010b). The present findings may therefore add to a body of 292 evidence suggesting a direct relationship between experience, increased holistic processing and face 293 processing efficiency (see also e.g. Crookes, Favelle \& Hayward, 2013; Degutis, Mercado, Wilmer \& 294 Rosenblatt, 2013; Susilo, Crookes, McKone \& Turner, 2009; Proietti, Pisacane \& Macchi Cassia, 2013).

295 Moreover, our results expand on these findings, as here a modulatory effect was related not to exposure 296 with a specific category of faces (own age, same-race, upright), but specific exemplars of the same 297 category.

\section{Conclusion}

To summarize, in keeping with the observation that personally familiar face identification is

300 robust across viewing distances (Ramon, 2015b) and therefore efficient even provided only low spatial

301 frequency information, we observed that familiarity was associated with a decreased reliance on local

302 details for discrimination of facial identity. Experience-related facilitation of perceptual processing was

303 found when global information was diagnostic for face discrimination (i.e., for dissimilar stimuli and

304 when visual information was degraded). Our findings demonstrate that individual face representations

305 obtained through real-life interactions and stored in memory enhance observers' ability to discriminate 306 identity-related information flexibly depending on the visual input available.

\section{Acknowledgements}

308 The authors express their gratitude to Bruno Rossion, under the supervision of which the experiments

309 were carried out. Further thanks are directed to all participants for their cooperation, as well as Matteo 
310 Visconti di Oleggio Castello and an anonymous reviewer for their constructive comments on an earlier

311 version of this manuscript.

\section{References}

313 Althoff RR \& Cohen NJ (1999). Eye-movement-based memory effect: a reprocessing effect in face

314 perception. J Exp Psychol Learn Mem Cogn, 25, 997-1010.

315 Bahrick HP, Bahrick PO \& Wittingler RP (1975). Fifty years of memory for names and faces: A cross316 sectional approach. J Exp Psychol Gen, 104, 54-75.

317 Barton JJ, Radcliffe N, Cherkasova MV, Edelman J \& Intriligator JM (2006). Information processing 318 during face recognition: the effects of familiarity, inversion, and morphing on scanning fixations. 319 Perception, 35, 1089-1105.

320 Beale JM \& Keil FC (1995). Categorical effects in the perception of faces. Cognition, 57, :217-39.

321 Brooks KR \& Kemp RI (2007). Sensitivity to feature displacement in familiar and unfamiliar faces: 322 beyond the internal/external feature distinction. Perception, 36, 1646-59.

323 Bruce V (1986). Influences of familiarity on the processing of faces. Perception ,15, 387-97.

324 Bruce V, Henderson Z, Greenwood K, Hancock P, Burton M \& Miller P (1999). Verification of face 325 identities from images captured on video. J Exp Psychol Appl, 5, 339-360.

326 Bruce V, Henderson Z, Newman C \& Burton AM (2001). Matching identities of familiar and unfamiliar 327 faces caught on CCTV images. J Exp Psychol Appl, 7, 207-18.

328 Bukach CM, Le Grand R, Kaiser MD, Bub DN \& Tanaka JW (2008). Preservation of mouth region 329 processing in two cases of prosopagnosia. J Neuropsychol, 2, 227-44.

330 Burton AM, Wilson S, Cowan M \& Bruce V (1999). Face recognition in poor quality video: evidence 331 from security surveillance. Psychological Science, 10, 243-8. 
332 Busigny T \& Rossion B (2010). Acquired prosopagnosia abolishes the face inversion effect. Cortex, 46, $333965-81$.

334 Busigny T, Bled C, Besson G \& Barbeau EJ (2012), The speed of face recognition: A 50ms gain between 335 personally familiar faces and famous faces. Perception 41 ECVP Abstract Supplement, page 20.

336 Busigny T, Graf M, Mayer E \& Rossion B (2010). Acquired prosopagnosia as a face-specific disorder: 337 ruling out the general visual similarity account. Neuropsychologia, 48, 2051-67.

338 Caharel S, Ramon M \& Rossion B (2014). Face familiarity decisions take $200 \mathrm{msec}$ in the human brain: 339 electrophysiological evidence from a go/no-go speeded task. J Cogn Neurosci, 26, 81-95.

340 Carbon CC (2008). Famous faces as icons. The illusion of being an expert in the recognition of famous 341 faces. Perception, 37, 801-806.

342 Collishaw SM \& Hole GJ (2000). Featural and configurational processes in the recognition of faces of 343 different familiarity. Perception, 29, 893-909.

344 Crookes K, Favelle S \& Hayward WG (2013). Holistic processing for other-race faces in chinese 345 participants occurs for upright but not inverted faces. Front Psychol, 4:29. doi: $346 \quad 10.3389 /$ fpsyg.2013.00029.

347 de Heering A \& Rossion B (2008). Prolonged visual experience in adulthood modulates holistic face 348 perception. PLoS One, 3(5):e2317.

349 Degutis J, Mercado RJ, Wilmer J \& Rosenblatt A (2013). Individual differences in holistic processing 350 predict the own-race advantage in recognition memory. PLoS One, 8(4):e58253.

351 Ellis HD, Shepherd JW \& Davies GM (1979). Identification of familiar and unfamiliar faces from internal 352 and external features: some implications for theories of face recognition. Perception, 8, 431-9. 
353 Fox CJ, Moon SY, Iaria G, Barton JJ (2009). The correlates of subjective perception of identity and 354 expression in the face network: an fMRI adaptation study. Neuroimage, 44:569-80.

355 Galton, F. (1883). Inquiries into human faculty and its development. London: Macmillan

356 Gilad-Gutnick S, Yovel G \& Sinha P (2012). Recognizing degraded faces: the contribution of configural 357 and featural cues. Perception, 41, 1497-511.

358 Gilaie-Dotan S \& Malach R (2007). Sub-exemplar shape tuning in human face-related areas. Cereb 359 Cortex, 17, 325-38.

360 Gobbini MI, Gors JD, Halchenko YO, Rogers C, Guntupalli JS, Hughes H \& Cipolli C (2013). Prioritized 361 Detection of Personally Familiar Faces. PLoS One, 21;8(6):e66620.

362 Goffaux V (2012). The discriminability of local cues determines the strength of holistic face processing. 363 Vision Research, 64:17-22.

364 Goffaux, V. \& Rossion, B. (2006). Faces are "spatial"--holistic face perception is supported by low spatial 365 frequencies. Journal of experimental psychology. Human perception and performance 32, 1023-1039.

366 Goffaux, V. (2009). Spatial interactions in upright and inverted faces: re-exploration of spatial scale 367 influence. Vision research, 49, 774-781

368 Hall, ET (1966). The Hidden Dimension. New York: Doubleday.

369 Jenkins R, White D, Van Montfort X \& Mike Burton A (2011). Variability in photos of the same face. 370 Cognition, 121, 313-23.

371 Jiang F, Blanz, V \& Rossion B. (2011). Holistic processing of shape cues in face identification: evidence 372 from face inversion, composite faces and acquired prosopagnosia. Visual Cognition, 19, 1003-1034.

373 Kuefner D, Macchi Cassia V, Picozzi M \& Bricolo E (2008). Do all kids look alike? Evidence for an 374 other-age effect in adults. J Exp Psychol Hum Percept Perform, 34, 811-7. 
375 Loftus GR \& Harley EM (2005). Why is it easier to identify someone close than far away? Psychon Bull 376 Rev, 12: 43-65.

377 McKone E, Kanwisher N \& Duchaine BC (2007). Can generic expertise explain special processing for 378 faces? Trends Cogn Sci, 11, 8-15.

Megreya AM \& Burton AM (2006). Unfamiliar faces are not faces: evidence from a matching task. Mem 380 Cognit, $34,865-76$.

381 382

Meyer DE, Irwin DE, Osman AM \& Kounios J (1988). The dynamics of cognition and action: mental processes inferred from speed-accuracy decomposition. Psychol Rev, 95, 183-237.

Michel C, Corneille O \& Rossion B (2007). Race categorization modulates holistic face encoding. Cogn Sci, 31, 911-24.Michel C, Rossion B, Han J, Chung CS \& Caldara R (2006). Holistic processing is finely tuned for faces of one's own race. Psychol Sci, 17, 608-15.

Mondloch C, Pathman T, Maurer D, Le Grand R \& de Schonen S (2007). The composite face effect in six-year-old children: Evidence of adult-like holistic face processing. Visual Cognition, 15, 564-577.

O'Donnell C \& Bruce V (2001). Familiarisation with faces selectively enhances sensitivity to changes made to the eyes. Perception, 30, 755-764.

Orban de Xivry, J.-J., Ramon, M., Lefèvre, P., Rossion, B. (2008). Reduced fixation on the upper area of personally familiar faces following acquired prosopagnosia. Journal of Neuropsychology, 2, 245-268.

Pachella RG (1974). The interpretation of reaction time in information-processing research. In B. H. Kantowitz (Ed.) Human information processing: Tutorials in performance and cognition. Hillsdale, N.J.: Lawrence Erlbaum Associates. Pp. 41-82.

Proietti V, Pisacane A \& Macchi Cassia V (2013) Natural Experience Modulates the Processing of Older Adult Faces in Young Adults and 3-Year-Old Children. PLoS ONE 8(2): e57499. 
397 Ramon M (2015). Differential processing of vertical interfeature relations due to real-life experience with 398 personally familiar faces. Perception, 44, 368-382.

399 Ramon M (2015). Perception of global facial geometry is modulated through experience. PeerJ. 2015 Mar $400 \quad$ 24;3:e850. doi: 10.7717/peerj.850. eCollection 2015.

401 Ramon M, Busigny T \& Rossion B (2010). Impaired holistic processing of unfamiliar individual faces in 402 acquired prosopagnosia. Neuropsychologia, 48, 933-44.

403 Ramon M, Busigny T, Gosselin G \& Rossion B (submitted). All new kids on the block? Impairment of 404 holistic processing of personally familiar faces in a kindergarten teacher with acquired prosopagnosia.

Ramon M, Caharel S \& Rossion B (2011). The speed of recognition of personally familiar faces. 406 Perception, 40, 437-449.

407

408

410

411

412

413

414

415

416

417

418

Ramon M, Dricot L, Rossion B (2010). Personally familiar faces are perceived categorically in faceselective regions other than the fusiform face area. Eur J Neurosci, 32: 1587-98.

Richler JJ \& Gauthier I (2013). When intuition fails to align with data: A reply to Rossion (2013). Visual Cognition, 21, 254-276.

Roberts T \& Bruce V (1989). Repetition priming of face recognition in a serial choice reaction time task. British Journal of Psychology, 80, 201-11.

Rossion B (2014). Understanding face perception by means of prosopagnosia and neuroimaging. Front Biosci (Elite Ed), Jun 1;6:258-307.

Rossion, B. (2013). The composite face illusion: a whole window into our understanding of holistic face perception. Visual Cognition, 21, 139-253.

Rotshtein P, Henson RN, Treves A, Driver J, Dolan RJ (2005). Morphing Marilyn into Maggie dissociates physical and identity face representations in the brain. Nat Neurosci, 8: 107-13. 
419 Russell R, Sinha P, Biederman I, Nederhouser M (2006). Is pigmentation important for face recognition?

420 Evidence from contrast negation. Perception, 35: 749-59.

421 Sadr J, Jarudi I \& Sinha, P (2003). The role of eyebrows in face recognition. Perception, 32, 285-293.

422 Schwaninger A, Wallraven C, Cunningham DW \& Chiller-Glaus SD (2006). Processing of facial identity 423 and expression: a psychophysical, physiological, and computational perspective. Prog Brain Res, 156, $424 \quad 321-43$.

425 426

434

Sergent J (1986). Microgenesis of face perception. In: Aspects of face processing. Eds. Ellis HD, Jeeves MA, Newcombe F, Young AM. Martinus Nijhoff, Dordrecht (1986).

Sergent, J. (1984). An investigation into component and configural processes underlying face perception. British Journal of Psychology, 75, 221-242.

Susilo T, Crookes K, McKone E \& Turner H (2009). The composite task reveals stronger holistic processing in children than adults for child faces. PLoS One; 4(7):e6460.

Tanaka, J. W., \& Farah, M. J. (2003). The holistic representation of faces. In M. A. Peterson \& G. Rhodes (Eds.), Perception of Faces, Objects, and Scenes: Analytic and Holistic Processes (pp. 53-74). New York, NY: Oxford University Press.

Tanaka,J. W., \& Farah, M. (1993). Parts and wholes in face recognition. Quarterly Journal of Experimental Psychology, 46, 225-245.

Tong F \& Nakayama K (1999). Robust representations for faces: evidence from visual search. J Exp Psychol Hum Percept Perform, 25, 1016-1035.

Van Belle G, Busigny T, Lefèvre P, Joubert S, Felician O, Gentile F \& Rossion B (2011). Impairment of holistic face percetion following right occipito-temporal damage in prosopagnosia: converging evidence from gaze-contingency. Neuropsychologia, 49, 3145-3150. 
441 Van Belle G, De Graef P, Verfaillie K, Busigny T \& Rossion B (2010). Whole not hole: expert face

442 recognition requires holistic perception. Neuropsychologia, 48, 2609-2620.

443 Van Belle G, De Graef P, Verfaillie K, Rossion B \& Lefèvre P (2010). Face inversion impairs holistic 444 perception: Evidence from gaze-contingent stimulation. Journal of Vision. May 1;10. pii: 10.5.10. doi:

445 10.1167/10.5.10.Van Belle G, Lefèvre P \& Rossion B (2012). Reduction of the perceptual field for 446 inverted faces:evidence from gaze contingency with full view stimuli. J Vis, 12(9): 625. 447 doi: $10.1167 / 12.9 .625$

448 Van Belle G, Lefèvre P, Laguesse R, Busigny T, De Graef P \& Verfaillie K (2010). Feature-based 449 processing of personally familiar faces in prosopagnosia: Evidence from eyegaze contingency. $450 \quad$ Behavioural Neurology, 23, 255-257.

451 Van Belle G, Ramon M, Lefèvre P \& Rossion B (2010). Fixation patterns during recognition of 452 personally familiar and unfamiliar faces. Front Psychol. 2010 Jun 17;1:20. doi: $453 \quad$ 10.3389/fpsyg.2010.00020. eCollection 2010.

454 Wang R, Li J, Fang H, Tian M \& Liu Jia (2012). Individual Differences in Holistic Processing Predict 455 Face Recognition Ability. Psychological Science, 23, 169-177.

456 Watier NN \& Collin CA (2009). Effects of familiarity on spatial frequency thresholds for face matching. 457 Perception, 38, 1497-507.Young AW, Hay DC, McWeeny KH, Flude BM \& Ellis AW (1985). 458 Matching familiar and unfamiliar faces on internal and external features. Perception, 14, 737-46. 


\section{Supplemental material}

461 Table S1 provides the accuracy scores and correct RTs per condition along with 95\%

462 bootstrapped confidence intervals. These were obtained per condition and group by randomly sampling

463 subjects with replacement; this process was repeated 999 times, leading to a distribution of bootstrapped

464 estimates of the mean accuracy and RT for each condition. Accuracy scores were considered above

465 chance and below ceiling if the confidence intervals did not contain .5 nor 1 , which was the case across

466 groups and experimental conditions.

467 Table S1. Average accuracy scores and RTs in msec along with 95\% bootstrap confidence intervals for each 468 condition.

\begin{tabular}{|c|c|c|c|c|}
\hline Group & Stimuli & Condition & $\begin{array}{l}\text { Accuracy } \\
{[95 \% \mathrm{CI}]}\end{array}$ & $\begin{array}{c}\text { RTs } \\
{[95 \% \mathrm{CI}]}\end{array}$ \\
\hline \multirow[t]{6}{*}{ Experimental } & \multirow[t]{3}{*}{ Familiar } & Full 20\% & $\begin{array}{c}.62 \\
{[.56 ; .67]}\end{array}$ & $\begin{array}{c}1056 \\
{[939 ; 1203]}\end{array}$ \\
\hline & & Full 50\% & $\begin{array}{c}.98 \\
{[.96 ; .99]}\end{array}$ & $\begin{array}{c}765 \\
{[689 ; 851]}\end{array}$ \\
\hline & & Blur 50\% & $\begin{array}{c}.89 \\
{[.82 ; .95]}\end{array}$ & $\begin{array}{c}881 \\
{[824 ; 944]}\end{array}$ \\
\hline & \multirow[t]{3}{*}{ Unfamiliar } & Full 20\% & $\begin{array}{c}.65 \\
{[.58 ; .72]}\end{array}$ & $\begin{array}{c}1062 \\
{[952 ; 1173]}\end{array}$ \\
\hline & & Full 50\% & $\begin{array}{c}.87 \\
{[.83 ; .91]}\end{array}$ & $\begin{array}{c}881 \\
{[812 ; 950]}\end{array}$ \\
\hline & & Blur 50\% & $\begin{array}{c}.66 \\
{[.59 ; .72]}\end{array}$ & $\begin{array}{c}910 \\
{[844 ; 978]}\end{array}$ \\
\hline \multirow[t]{6}{*}{ Control } & \multirow[t]{3}{*}{ Familiar } & Full 20\% & $\begin{array}{c}.77 \\
{[.73 ; .81]}\end{array}$ & $\begin{array}{c}994 \\
{[878 ; 1118]}\end{array}$ \\
\hline & & Full 50\% & $\begin{array}{c}.92 \\
{[.89 ; .95]}\end{array}$ & $\begin{array}{c}813 \\
{[717 ; 933]}\end{array}$ \\
\hline & & Blur 50\% & $\begin{array}{c}.79 \\
{[.75 ; .83]}\end{array}$ & $\begin{array}{c}869 \\
{[774 ; 993]}\end{array}$ \\
\hline & \multirow[t]{3}{*}{ Unfamiliar } & Full 20\% & $\begin{array}{c}.77 \\
{[.71 ; .82]}\end{array}$ & $\begin{array}{c}954 \\
{[807 ; 1126]}\end{array}$ \\
\hline & & Full 50\% & $\begin{array}{c}.89 \\
{[.85 ; .93]}\end{array}$ & $\begin{array}{c}883 \\
{[747 ; 1052]}\end{array}$ \\
\hline & & Blur $50 \%$ & $\begin{array}{c}.70 \\
{[.63 ; .76]}\end{array}$ & $\begin{array}{c}907 \\
{[790 ; 1033]}\end{array}$ \\
\hline
\end{tabular}




\section{Figure 1 (on next page)}

\section{Figure 1}

Stimuli discriminated in the delayed matching task. a. An example of a familiar face morph continuum. Unfamiliar stimuli were those created and used in a previous study (see Ramon et al., 2010 for examples and details regarding stimulus creation), which were unfamiliar to all subjects tested. Familiar morph continua were created from pairs of classmates of experimental subjects tested, and were unfamiliar to control subjects. b. Examples of stimulus pairs to be discriminated in the 2AFC delayed matching task (pairs were taken from either side of a continuum). 
a.

$100 \%$ Face A $\quad$ PeerJ $\quad$ Manuscript to be reviewed

b.

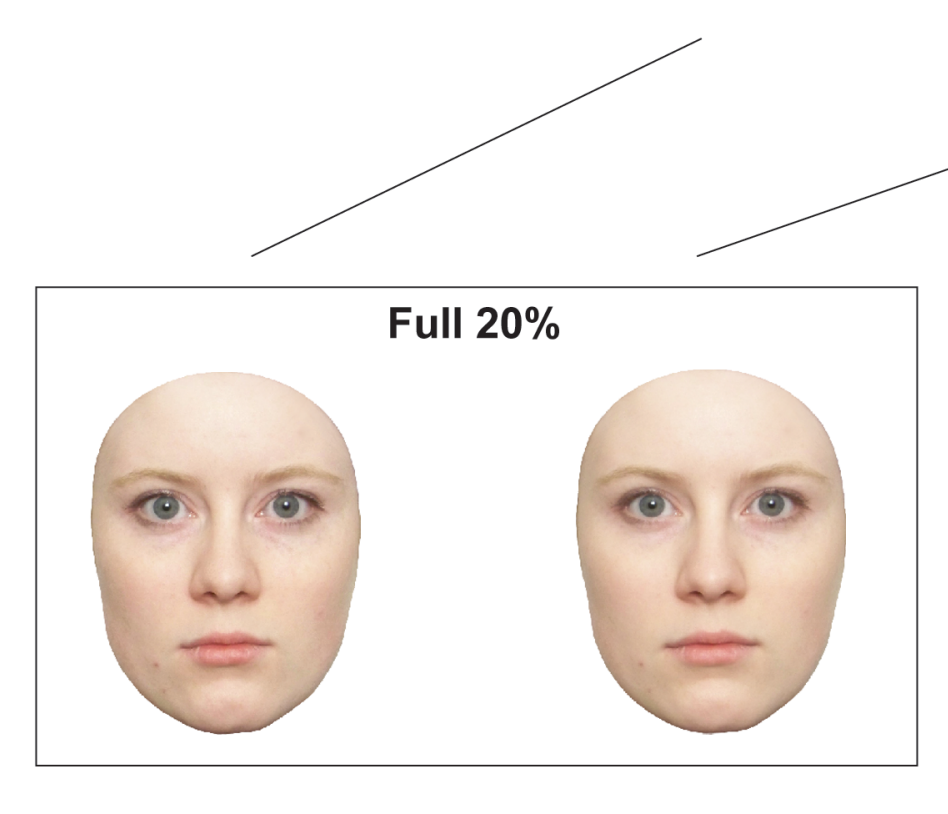

Blur 50\%
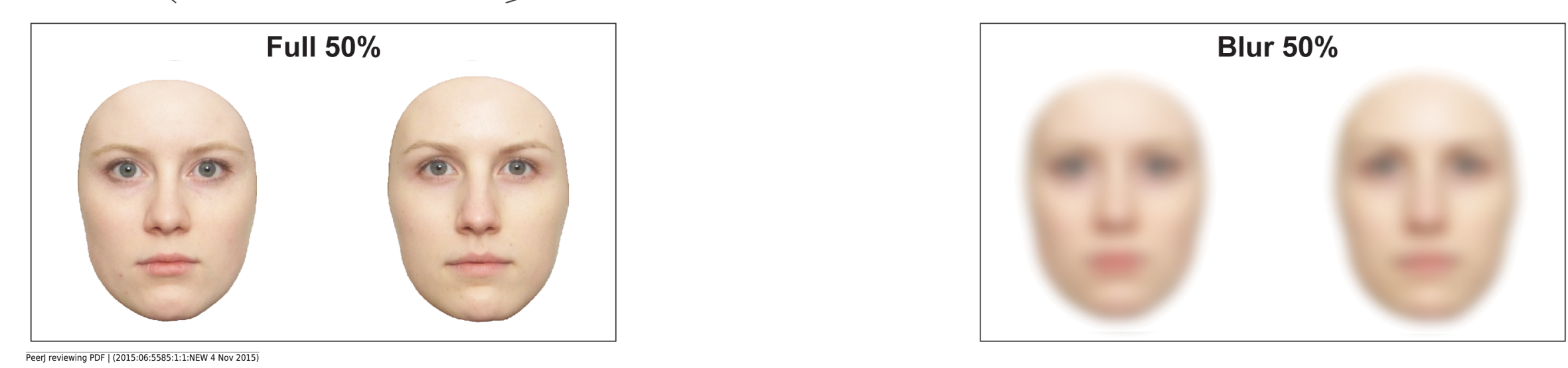


\section{Figure 2 (on next page)}

\section{Figure 2}

Familiarity advantage in the 2AFC delayed matching task with personally familiar and unfamiliar morph stimuli. Mean familiarity advantage ((familiar - unfamiliar) / (familiar + unfamiliar)) for accuracy scores per condition observed for control, as well as experimental subjects. Error bars represent standard errors for both measures. Note that for control subjects, all faces presented were unfamiliar. 


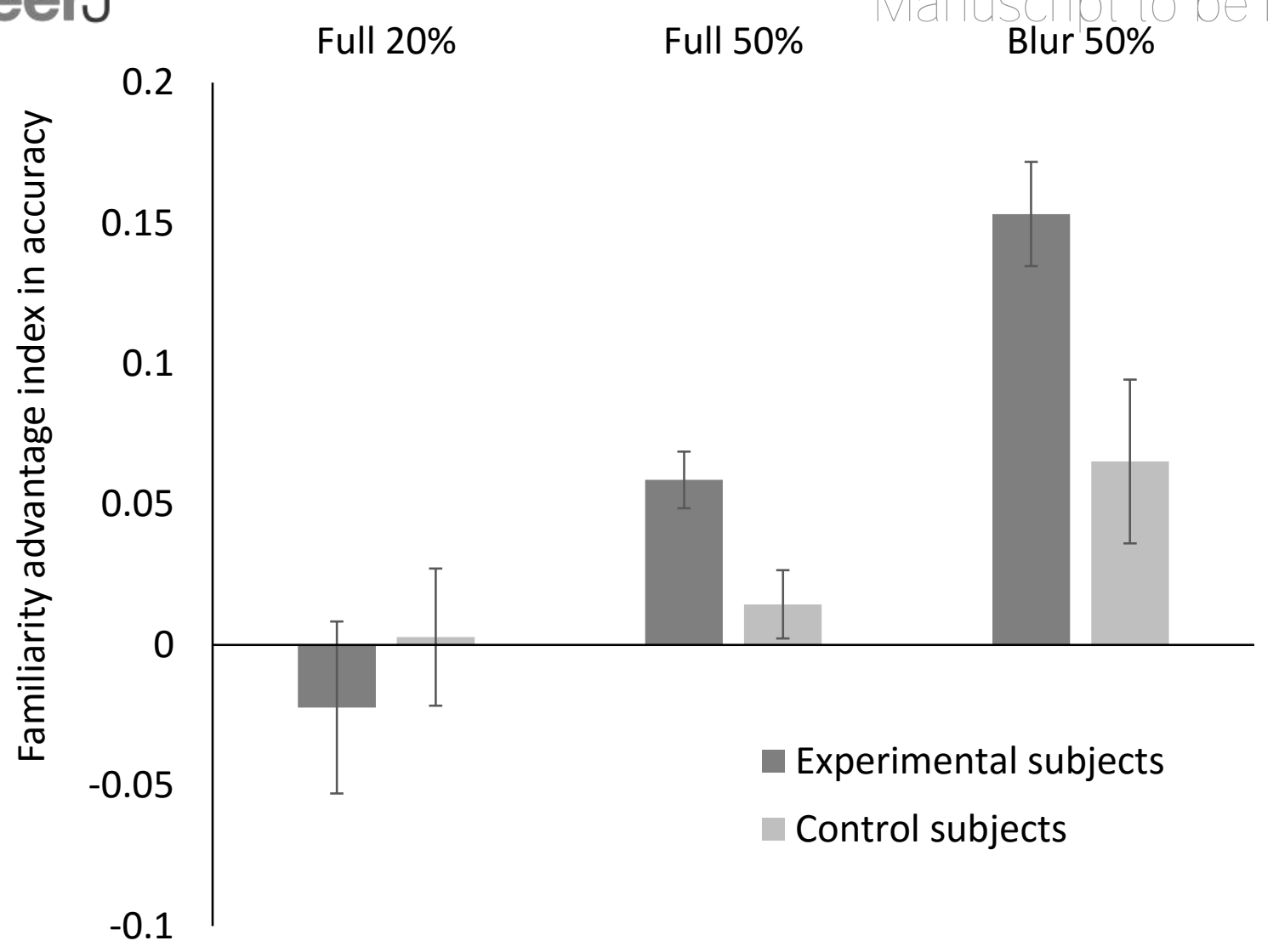


